\title{
Polarization tensors in strong magnetic fields
}

AUTHOR(S):

Kohri, K; Yamada, S

CITATION:

Kohri, K ...[et al]. Polarization tensors in strong magnetic fields.

PHYSICAL REVIEW D 2002, 65(4): 043006.

ISSUE DATE:

2002-02-15

URL:

http://hdl.handle.net/2433/50453

RIGHT:

Copyright 2002 American Physical Society 
PHYSICAL REVIEW D, VOLUME 65, 043006

\title{
Polarization tensors in strong magnetic fields
}

\author{
Kazunori Kohri \\ Yukawa Institute for Theoretical Physics, Kyoto University, Kyoto, 606-8502, Japan \\ Shoichi Yamada \\ Institute of Laser Engineering (ILE), Osaka University, Osaka 565-0871, Japan
}

(Received 14 February 2001; revised manuscript received 9 November 2001; published 23 January 2002)

\begin{abstract}
The vacuum polarization tensor in strong external magnetic fields has been evaluated numerically for various strengths of magnetic fields and momenta of photons under the threshold of the $e^{ \pm}$pair creation. The fitting formula has been obtained which reproduces the calculated results within $10 \%$ of error. The proper time method is employed further to obtain the retarded polarization tensor for finite temperature plasmas.
\end{abstract}

DOI: 10.1103/PhysRevD.65.043006

PACS number(s): 97.60.Gb, 12.20.Ds, 98.70.Rz

\section{INTRODUCTION}

The strong magnetic field is attracting the attention of astrophysicists these days. It has been known that the magnetized vacuum shows interesting features as the magnetic field strength exceeds a critical value $B_{c}=m_{e}^{2} / e \sim 4$ $\times 10^{13} \mathrm{G}[1,2]$. Since this value is so large even in the universe compared, for example, with the canonical magnetic field of $10^{12} \mathrm{G}$ for a pulsar, it was supposed that this was a subject of academic interest only. This has been changing drastically recently.

Some observations [3] suggest the existence of neutron stars with a magnetic field far greater $\left(\sim 10^{15} \mathrm{G}\right)$ than the canonical one for the observed pulsars, and they are called a magnetar, as dubbed by Duncun and Thompson [4]. As the reality of very large magnetic fields looms, some researchers speculated further that some other peculiar extraterrestrial phenomena might also involve very strong magnetic fields. Among them are gamma ray bursts and hypernovae [5]. It is supposed for the latter that a jet is somehow produced in these objects and the dipolar magnetic field is playing an essential role. On the other hand, some models for the gamma ray bursts are employing the magnetic fields to extract the enormous energy of the phenomenon itself [6]. Furthermore, evidence of generic asymmetry for collapse-driven supernovae has been accumulated [7], and the strong magnetic field might have some implications for the ordinary supernova if the magnetar as observed is the end product of the supernova explosion and the observed asymmetry is a dynamical consequence involving the strong magnetic field [8]. The fast proper motion of young pulsars might be explained by the combination of the strong magnetic fields and some processes such as neutrino oscillations, for example [9]. The explosion mechanism of the supernova will be changed substantially as well as nucleosynthesis therein.

It is, therefore, not only of academic interest to consider the features of the strongly magnetized vacuum. In particular, the quantum electrodynamical processes are most important, since those objects quoted above are mostly observed by electromagnetic waves, though the weak interactions are no less important $[9,10]$. The study of the strongly magnetized vacuum has a long history, though. Adler [11], for example, gave detailed formulations for the polarization tensor and the photon splitting rate as well as some useful analytic expressions for limit cases (see also [2,12-14] and the references therein for many other contributions). Astrophysicists have used these approximate formulas for their model building $[15,16]$.

Those analytic expressions are approximate ones, though, valid for some limit cases such as the strong or weak magnetic field limits and the zero photon energy limit. It appears that we are lacking the complementary numerical evaluations of the polarization tensor for the intermediate values of magnetic field strength and/or photon energy. In particular, the energy dependence near the threshold of the pair creation has not been studied in detail. It is the purpose of the paper to fill this gap and give the interpolation formula based on the fitting to the numerical integrations. Our interest is, however, also directed to the dispersion relation of photons in the strongly magnetized plasma. In Sec. IV we will extend the Schwinger formulation and give the expression for the retarded polarization tensor for finite temperature plasmas.

The paper is organized as follows. In Sec. II we formulate the vacuum polarization tensors in a strong magnetic field. We study the refractive indices numerically in Sec. III. In Sec. IV we extend the formulation to the retarded polarization tensors in the finite density and temperature cases. Finally, Sec. V is devoted to the conclusion.

\section{VACUUM POLARIZATION IN A STRONG MAGNETIC FIELD}

This section is devoted to giving the formula of the vacuum polarization tensor which we employ from the previous papers $[12,13]$ for the numerical calculations. Using Schwinger's proper-time method [17], as was shown in Ref. [13], the vacuum polarization tensor in a strong magnetic field (see Fig. 1) is expanded as

$$
\Pi^{\mu \nu}(k, B)=\sum_{i=0}^{2} G_{i} f_{i}^{\mu \nu},
$$

where $k=\left(\omega, k_{\perp}, 0, k_{\|}\right)$is the energy-momentum 4-vector of a photon, $\omega$ the photon energy, $k_{\perp}=\omega \sin \theta, k_{\|}=\omega \cos \theta, \theta$ 


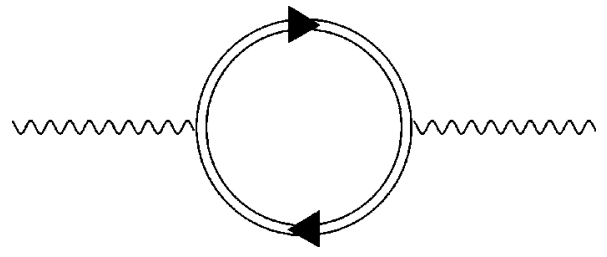

FIG. 1. Vacuum polarization in a strong magnetic field. The double solid line denotes the electron propagator which includes all contributions of the vertices from the magnetic field.

the direction of 3-momentum with respect to the magnetic field, and $B$ is the magnetic field. $G_{i}$ is given by the following equations:

$$
G_{i}=\frac{e^{2}}{(2 \pi)^{2}} L \int_{0}^{\infty} d \alpha \int_{0}^{1} d \beta \gamma_{i} E(\alpha, \beta, k, L),
$$

with

$$
\begin{aligned}
\gamma_{0}= & k^{2} \frac{\cosh (L \beta \alpha)}{2 \sinh (L \alpha)}\left\{1-\beta \frac{\tanh (L \beta \alpha)}{\tanh (L \alpha)}\right\}, \\
\gamma_{1}= & k_{\perp}^{2}\left[\frac{\cosh (L \beta \alpha)-\cosh (L \alpha)}{\sinh ^{3}(L \alpha)}\right. \\
& +\frac{\cosh (L \beta \alpha)}{2 \sinh (L \alpha)}\left\{1-\beta \frac{\tanh (L \beta \alpha)}{\tanh (L \alpha)}\right\}, \\
\gamma_{2}= & \left(\omega^{2}-k_{\|}^{2}\right)\left[\frac{1-\beta^{2}}{2} \operatorname{coth}(L \alpha)\right. \\
& -\frac{\cosh (L \beta \alpha)}{2 \sinh (L \alpha)}\left\{1-\beta \frac{\tanh (L \beta \alpha)}{\tanh (L \alpha)}\right\},
\end{aligned}
$$

and

$$
\begin{aligned}
E(\alpha, \beta, k, L)= & \exp \left[-\alpha+\frac{\alpha\left(1-\beta^{2}\right)}{4} \frac{k^{2}}{m_{e}^{2}}+\left\{\frac{\alpha\left(1-\beta^{2}\right)}{4}\right.\right. \\
& \left.\left.+\frac{\cosh (L \beta \alpha)-\cosh (L \alpha)}{2 L \sinh (L \alpha)}\right\} \frac{k_{\perp}^{2}}{m_{e}^{2}}\right],
\end{aligned}
$$

where $e$ denotes the electron charge in MKS units $\left(e^{2}\right.$ $\simeq 1 / 137), m_{e}$ is the electron mass, and $L\left(\equiv B / B_{c}\right)$ is a dimensionless magnetic field normalized by the critical magnetic field $\left(B_{c}=m_{e}^{2} / e\right) . f_{i}^{\mu \nu}$ is expressed as

$$
\begin{aligned}
& f_{0}^{\mu \nu}=g^{\mu \nu}-\frac{k^{\mu} k^{\nu}}{k^{2}}, \\
& f_{l}^{\mu \nu}=\frac{b_{l}^{\mu} b_{l}^{\nu}}{b_{l}^{\gamma} b_{l \gamma}} \quad(\text { for } l=1,2),
\end{aligned}
$$

with

$$
\begin{aligned}
& b_{1}^{\mu}=F^{\mu \gamma} k_{\gamma}, \quad b_{2}^{\mu}=F^{* \mu \gamma} k_{\gamma}, \\
& b_{3}^{\mu}=k^{2} F^{\mu \gamma} F_{\gamma \delta} k^{\delta}-k^{\mu} k^{\gamma} F_{\gamma \delta} F^{\delta \epsilon} k_{\epsilon}, \\
& b_{4}^{\mu}=k^{\mu}
\end{aligned}
$$

where $F^{\mu \nu}$ denotes the Maxwell stress 4-tensor and its dual tensor is defined by $F^{* \mu \nu} \equiv-\frac{1}{2} \epsilon^{\mu \nu \gamma \delta} F_{\gamma \delta}$.

As was carefully discussed in Ref. [13], only $G_{0}$ contributes to the vacuum polarization tensor if the magnetic field is very weak, $L \ll 1$. In such a week field limit, we know the form of $\Pi^{\mu \nu}(k)$ in familiar QED as

$$
\Pi^{\mu \nu}(k, 0)=\left.G_{0}\right|_{B=0} f_{0}^{\mu \nu},
$$

where

$$
\begin{aligned}
\left.G_{0}\right|_{B=0}= & \frac{e^{2}}{(2 \pi)^{2}} k^{2} \int_{0}^{\infty} d \alpha \int_{0}^{1} d \beta \frac{1-\beta^{2}}{2 \alpha} \\
& \times \exp \left[-\alpha+\frac{\alpha\left(1-\beta^{2}\right)}{4} \frac{k^{2}}{m_{e}^{2}}\right] .
\end{aligned}
$$

In Eq. (13) we should get rid of the divergence at $\alpha=0$ and regularize it. Then we obtain the regularized form of the vacuum polarization tensor at $L=0$,

$$
\operatorname{reg} \Pi^{\mu \nu}(k, 0)=A(k) f_{0}^{\mu \nu},
$$

with

$$
\begin{aligned}
A(k) & \left.\equiv \operatorname{reg} G_{0}\right|_{B=0} \\
& =-\frac{e^{2}}{(2 \pi)^{2}} k^{2}\left[\frac{1}{9}-\frac{(1-h(k))\left(4 m_{e}^{2}+2 k^{2}\right)}{3 k^{2}}\right],
\end{aligned}
$$

where

$$
h(k)= \begin{cases}\sqrt{\frac{4 m_{e}^{2}}{k^{2}}-1 \cot ^{-1}\left(\sqrt{\frac{4 m_{e}^{2}}{k^{2}}-1}\right)} \quad & \left(\text { for } 0 \leqslant k^{2} \leqslant 4 m_{e}^{2}\right), \\ \sqrt{1-\frac{4 m_{e}^{2}}{k^{2}}} \operatorname{coth}^{-1}\left(\sqrt{1-\frac{4 m_{e}^{2}}{k^{2}}}\right) & \left(\text { for } k^{2}<0\right) .\end{cases}
$$


Thus, to obtain the regularized form of the polarization tensor in a strong magnetic field ( $L \gtrsim 1)$, we have only to substitute $G_{0}$ with

$$
\operatorname{reg} G_{0}=G_{0}-\left.G_{0}\right|_{B=0}+A(k) .
$$

As we mentioned in the previous sections, the refractive indices of photon would deviate from unity in a strong magnetic field because the vacuum polarization is influenced by the magnetic field and the dispersion relation is changed. The refractive indices are defined from the dispersion relation as

$$
\mu^{2}=\frac{|\boldsymbol{k}|^{2}}{\omega^{2}}
$$

where $\boldsymbol{k}$ is a spatial 3-vector of $k$. To obtain the dispersion relation in a strong magnetic field, we consider the wave equation of the photon,

$$
\left[k^{2} g^{\mu \nu}-k^{\mu} k^{\nu}+4 \pi \operatorname{reg} \Pi^{\mu \nu}(k)\right] A_{\mu}(k)=0 .
$$

In this equation the prefactor of $\Pi^{\mu \nu}(k)$, " $4 \pi$," originates in MKS unit. The determinant of the matrix should be zero so that Eq. (19) could have a nontrivial solution. When we choose the radiation gauge $A_{\mu}=(0, \boldsymbol{A})$, we get a quadratic equation and we obtain two solutions,

$$
\begin{aligned}
& \mu_{1}^{2}=\frac{1+\chi_{0}}{1+\chi_{0}-\sin ^{2} \theta \chi_{1}}, \\
& \mu_{2}^{2}=\frac{1+\chi_{0}+\chi_{2}}{1+\chi_{0}+\cos ^{2} \theta \chi_{2}},
\end{aligned}
$$

where

$$
\begin{aligned}
& \chi_{0}=\frac{4 \pi \mathrm{reg} G_{0}}{k^{2}}, \\
& \chi_{1}=\frac{4 \pi G_{1}}{k_{\perp}^{2}}, \\
& \chi_{2}=\frac{4 \pi G_{2}}{k_{\|}^{2}} .
\end{aligned}
$$

Here $\mu_{1}^{2}$ corresponds to the eigen vector $(0,1,0)$ and $\mu_{2}^{2}$ to $\left(\left(1+\chi_{0}+\chi_{2}\right) \cos \theta, 0,-\left(1+\chi_{0}\right) \sin \theta\right)$. Since each $\chi_{i}$ depends on $\mu^{2}$ through $k$ and $G_{i}$, Eqs. (20) and (21) are implicit equations for $\mu^{2}$. In the literature, e.g., [13], the authors gave only the integral form and some limit cases.

To wrap up, we numerically integrate Eq. (2) and then regularize the results according to Eq. (17). Using this result, we solve the implicit Eqs. (20) and (21) to obtain the refractive indices.

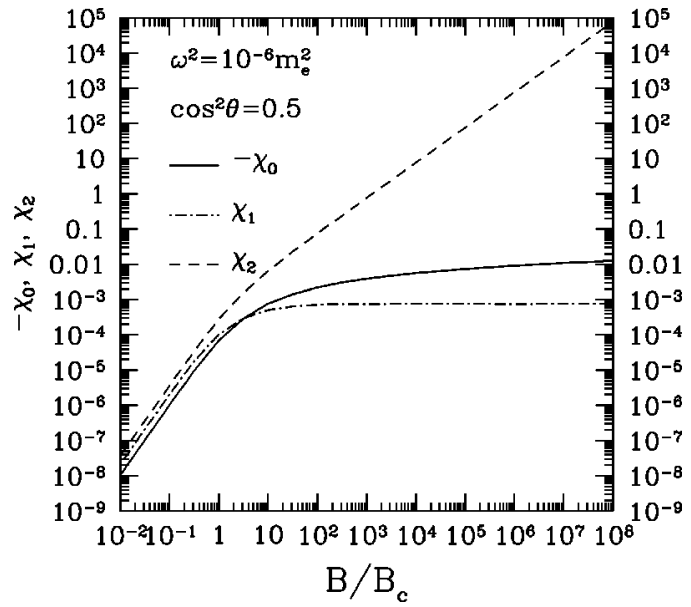

FIG. 2. Plots of $-\chi_{0}, \chi_{1}$, and $\chi_{2}$ as a function of $B / B_{c}$. Here we adapt $\omega^{2}=10^{-6} \mathrm{~m}_{e}^{2}$ and $\cos ^{2} \theta=0.5$. We find that the magnitude of $\chi_{0}$ increases as $B / B_{c}$ increases $\left[\propto \log \left(B / B_{c}\right)\right], \chi_{1}$ reaches the limit value $\left(\sim 7.7 \times 10^{-4}\right)$, and $\chi_{2} \simeq\left(e^{2} / 3 \pi\right) B / B_{c}$ for $B / B_{c} \gg 1$.

\section{NUMERICAL RESULTS FOR THE VACUUM POLARIZATION TENSOR IN A STRONG MAGNETIC FIELD}

We show here the main results for the magnetized vacuum. The numerical integrations of Eq. (2) have been done with the Monte Carlo method in the parameter region covering a wide range of the magnetic field strength, the direction of the photon propagation and the photon energy below the threshold of the pair creation. In limiting cases, i.e., both in the large and small $B$ limits, in advance we also calculated the $\chi_{i} \mathrm{~s}$ analytically. Comparing with the analytical esimations, we can check the validities in the numerical results.

In Fig. 2 we plot $-\chi_{0}$ as a function of $L\left(=B / B_{c}\right)$ in a low energy limit $\left(\omega^{2}=10^{-6} m_{e}^{2}\right)$. From the plot, we find that the magnitude of $\chi_{0}$ increases as $L$ increases. Thus the contribution from $\chi_{0}$ to the refractive indices in Eqs. (20) would not be negligible in an extremely strong magnetic field. ${ }^{1}$ This behavior agrees with our analytical estimation that $\chi_{0} \propto$ $-\log (L)$ in a strong magnetic field limit $(L \rightarrow \infty)$ and contradicts the statement by Melrose and Stoneham [13] that $\chi_{0}$ $\propto \exp [-L]$ for this limit. This difference, however, is substantial only for extremely large magnetic fields. Nonetheless, we did not drop this term in estimating the correct refractive indices. In Fig. 2 we also plot $\chi_{1}$ and $\chi_{2}$ as a function of $L . \chi_{1}$ approaches the limit value $\left(\sim 7.7 \times 10^{-4}\right)$ for $L \gg 1$. This feature is consistent with our analytical estimation and again disagrees with Melrose and Stoneham [13]

\footnotetext{
${ }^{1}$ Here we assume the one-loop approximation. Since the dependence of the polarization tensor on the strength of the magnetic field is complicated even in this approximation after taking full account of the external magnetic field by Schwinger's proper time method, it is a nontrivial issue whether the higher-loop corrections dominate over the one-loop contribution in the strong magnetic field limit and above what strength of the magnetic field even if they do become dominant.
} 


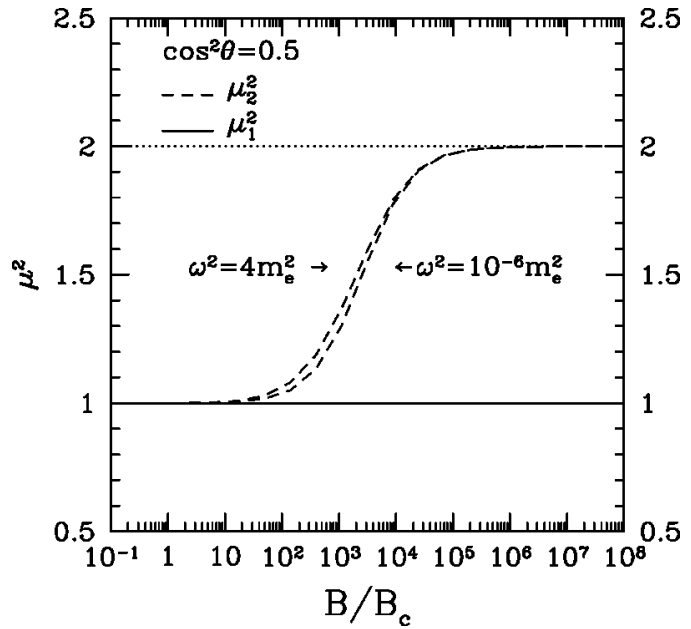

FIG. 3. Plot of the refractive indices as a function of $B / B_{c}$. The solid line represents $\mu_{1}^{2}$ and the dashed line represents $\mu_{2}^{2}$. The left (right) dashed line is the case for $\omega^{2}=4 m_{e}^{2}\left(10^{-6} m_{e}^{2}\right)$. Here we adapt $\omega^{2}=10^{-6} m_{e}^{2}$ and $\cos ^{2} \theta=0.5$. We find that $\mu_{2}^{2}$ reaches the limit value $\left(1 / \cos ^{2} \theta=2\right)$ in the strong $B$ limit.

who claimed that $\chi_{1} \propto \exp [-L]$ for this limit. As for $\chi_{2}$, it is found that $\chi_{2}$ is linearly proportional to $L$ in the strong magnetic field and the weak energy limit. It agrees exactly with our analytical estimation that $\chi_{2} \simeq\left(e^{2} / 3 \pi\right) L$ under the condition that $L \gg 1, \omega^{2}-k_{\|}^{2} \ll 4 m_{e}^{2}$, and $k_{\perp}^{2} \ll 2 m_{e}^{2} L[13]$.

In addition, in the case of weak magnetic-field and low energy limits, i.e., $L \ll 1$ and $\omega \ll m_{e}$, the numerical result of the behaviors of $\chi_{0}, \chi_{1}$, and $\chi_{2}$ also agrees with the analytical estimations,

$$
\begin{gathered}
-\chi_{0} \rightarrow \frac{2 e^{2}}{45 \pi} L^{2} \simeq 1.03 \times 10^{-4} L^{2}, \\
\chi_{1} \rightarrow \frac{4 e^{2}}{45 \pi} L^{2} \simeq 2.07 \times 10^{-4} L^{2}, \\
\chi_{2} \rightarrow \frac{7 e^{2}}{45 \pi} L^{2} \simeq 3.61 \times 10^{-4} L^{2} .
\end{gathered}
$$

In Fig. 3 we plot the obtained refractive indices as a function of $L$. The solid and dashed lines represent $\mu_{1}^{2}$ and $\mu_{2}^{2}$, respectively. It is easy for us to understand the behavior of $\mu^{2}$ 's in a strong magnetic field. From Eqs. (20) and (21), we find that

$$
\begin{aligned}
& \mu_{1}^{2} \simeq 1+7.7 \times 10^{-4} \sin ^{2} \theta, \\
& \mu_{2}^{2} \simeq 1 / \cos ^{2} \theta,
\end{aligned}
$$

for $L \gg 3 \pi / \alpha$ in the case of the weak energy and as long as $\chi_{0}$ is much smaller than unity. The photon-energy dependence of $\mu^{2}$ is shown in Fig. 4. In this plot we find that near the threshold $\left(\omega^{2} \sim 4 m_{e}^{2}\right)$ the energy dependence of $\mu_{2}^{2}$ becomes important. Note that this behavior has not been studied so far. The result suggests that the refractive index and the polarization tensor as well will have a nontrivial feature

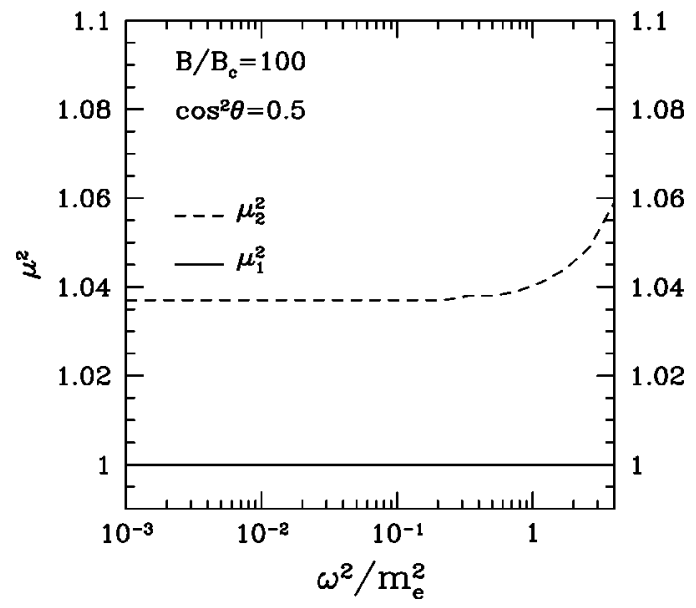

FIG. 4. Plot of the refractive indices as a function of $\omega^{2} / m_{e}^{2}$. The solid line represents $\mu_{1}^{2}$ and the dashed line represents $\mu_{2}^{2}$. Here we adapt $B / B_{c}=10^{2}$ and $\cos ^{2} \theta=0.5$.

above the threshold energy and that their numerical evaluations are required there also. $\mu_{1}^{2}$ is insensitive to the photon energy, which justifies the neglect of its energy dependence in the analyses so far. In Fig. 5 we plot $\mu^{2}$ as a function of $\cos ^{2} \theta$ in a strong magnetic field $\left(L=10^{8}\right)$. It is clear that $\mu_{2}^{2}$ is proportional to $1 / \cos ^{2} \theta$ which is analytically expected in Eq. (29).

Here we give the fitting formula of $\mu_{2}^{2}$,

$$
\mu_{2}^{2}=C_{1}\left\{\tanh \left[C_{2}\left(x-C_{3}\right)\right]+1\right\}+1,
$$

with

$$
x=\log _{10}\left(B / B_{c}\right),
$$

and

$$
C_{1}=\tan ^{2} \theta / 2
$$

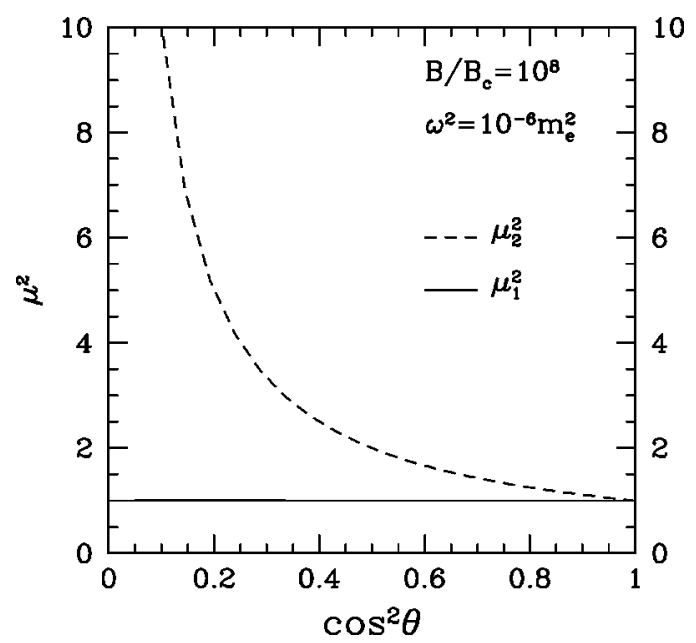

FIG. 5. Plot of the refractive indices as a function of $\cos ^{2} \theta$. The solid line represents $\mu_{1}^{2}$ and the dashed line represents $\mu_{2}^{2}$. Here we adapt $B / B_{c}=10^{8}$ and $\omega^{2}=10^{-6} \mathrm{~m}_{e}^{2}$. We find that $\mu_{2}^{2}$ scales $1 / \cos ^{2} \theta$ in such a strong $B$ limit. 


$$
\begin{aligned}
& C_{2}=1.15-7.07 \times 10^{-3}\left(\omega^{2} / m_{e}^{2}\right)^{1.60} / \sqrt{\cos ^{2} \theta}, \\
& C_{3}=3.11-\log _{10}\left(\cos ^{2} \theta\right)-1.84 \times 10^{-2}\left(\omega^{2} / m_{e}^{2}\right) / \sqrt{\cos ^{2} \theta} .
\end{aligned}
$$

This fitting formula reproduces the numerical results within the error of less than $10 \%$ for a wide parameter range $(0.1$ $\leqslant \cos ^{2} \theta \leqslant 1, \omega^{2} / m_{e}^{2} \leqslant 4$, and $\left.0 \leqslant B / B_{c} \lesssim 10^{10}\right)$. In the large $L$ limit especially, Eq. (30) approaches the value of the analytical estimation $\left(\simeq 1 / \cos ^{2} \theta\right)$. Of course, the small $L$ limit reproduces $\mu_{2}^{2}=1$ exactly. In addition, the low energy limit of the fitting formula $\left(\omega^{2} \rightarrow 0\right)$ agrees with the numerical estimations very well within less than $1 \%$.

Because it seems that concrete values of the polarization tensor near the threshold energy of the pair creation have never been given in the literature, we hope that the above results would serve as a bridge between the analytic formulas given for the low energy limit [11]. Although our results are confined only below the threshold energy of pair creations, we think those still contain interesting parameter regions from the point of view of astrophysics, e.g., x-ray observations $(E=\mathrm{a}$ few $\mathrm{keV}-100 \mathrm{keV})$. After the discovery of the magnetars, it is not unimaginable that astrophysical objects with yet stronger magnetic fields will be found. In fact, the magnetic field in the core of a rapidly rotating supernova might reach $\sim 10^{17} \mathrm{G}$ or more. In such superstrong magnetic fields, we expect that there would exist non-trivial lensing effects in $\mathrm{x}$ rays due to the modifications of the refractive indices. Therefore, we think that astrophysicists might have to consider such lensing effects there for $\mathrm{x}$-ray observations [18].

In addition, as mentioned above, our results suggest that there will be nontrivial features in the polarization tensor above the threshold energy. The numerical study not only of the real part but also of the imaginary part of the polarization tensor above the threshold will be studied in a separate paper [19].

\section{RETARDED POLARIZATION TENSOR IN FINITE TEMPERATURE PLASMAS}

In the previous sections we have considered the vacuum polarization tensor. It is also our concern to calculate the polarization tensor for plasmas with finite temperatures. We will extend the previous formulation to the finite density and temperature case. We will rely on the real time formalism of the finite density and temperature field theory and obtain the expression for the retarded polarization tensor. One advantage of the formulation employed for the vacuum polarization Sec. III is the fact that it avoids the summation over the Landau levels. This can be achieved also for the finite density and temperature case as shown below. Recently, some authors $[20,21]$ gave the expression of the chronological polarization tensor on a similar footing. Their main concern is the behavior of the polarization tensor in the weak magnetic field limit and the final formula is a five-dimensional integration. Although two polarizations are related to each other, the retarded one allows more direct physical interpretation. Moreover, the retarded polarization tensor should be ob- tained by analytical extension of the imaginary time polarization tensor which has been given by the authors [22-24].

Since the spectral density is unchanged by the introduction of the heat bath, the polarization tensor for the finite density and temperature can be obtained from the vacuum polarization tensor under the same approximation. It is known from the finite density and temperature field theory that the retarded polarization tensor is expressed as

$$
\begin{aligned}
i \Pi_{r}^{\mu \nu}\left(x, x^{\prime}\right)= & \frac{e^{2}}{2} \operatorname{Tr}\left[\gamma^{\mu} G_{c}\left(x, x^{\prime}\right) \gamma^{\nu} G_{a}\left(x^{\prime}, x\right)\right. \\
& \left.+\gamma^{\mu} G_{r}\left(x, x^{\prime}\right) \gamma^{\nu} G_{c}\left(x^{\prime}, x\right)\right] .
\end{aligned}
$$

In the above equation, the subscripts $r, a, c$ denote the retarded, advanced, and correlation components of the Green function, respectively [25]. For the stationary system, we can in general assume that the vector potential is time independent. In this case the polarization tensor $\Pi_{r}\left(x, x^{\prime}\right)$ is a function of the time difference $t-t^{\prime}$ alone. It is possible that it depends on the spatial coordinates $\mathbf{x}$ and $\mathbf{x}^{\prime}$ separately. Fourier transforming the polarization tensor with respect to $t$ $-t^{\prime}$, we obtain

$$
\begin{aligned}
i \widetilde{\Pi}_{r}\left(p_{0}\right)= & \frac{e^{2}}{2} \int \frac{d q_{0}}{2 \pi} \operatorname{Tr}\left[\gamma^{\mu} \widetilde{G}_{c}\left(q_{0}+p_{0}\right) \gamma^{\nu} \widetilde{G}_{a}\left(q_{0}\right)\right. \\
& \left.+\gamma^{\mu} \widetilde{G}_{r}\left(q_{0}+p_{0}\right) \gamma^{\nu} \widetilde{G}_{c}\left(q_{0}\right)\right] .
\end{aligned}
$$

In the above equation, the tilde means the Fourier component and the spatial coordinates are dropped for simplicity. The correlation component of the Green function in the thermal equilibrium can be expressed by the retarded and advanced Green functions and the distribution function by the following relation:

$$
\widetilde{G}_{c}\left(p_{0}\right)=\widetilde{G}_{r}\left(p_{0}\right)\left[1-2 f\left(p_{0}\right)\right]-\left[1-2 f\left(p_{0}\right)\right] \widetilde{G}_{a}\left(p_{0}\right) .
$$

Noting that the retarded and advanced Green functions for the ideal electrons of finite density and temperature are identical to the counterparts for vacuum, we obtain

$$
\begin{aligned}
& \widetilde{G}_{r}\left(q_{0}\right)=\Theta\left(q_{0}\right) \widetilde{G}_{F}\left(q_{0}\right)-\Theta\left(-q_{0}\right) \widetilde{G}_{\widetilde{F}}\left(q_{0}\right) \\
& \widetilde{G}_{a}\left(q_{0}\right)=\Theta\left(-q_{0}\right) \widetilde{G}_{F}\left(q_{0}\right)-\Theta\left(q_{0}\right) \widetilde{G}_{\widetilde{F}}\left(q_{0}\right) .
\end{aligned}
$$

The subscripts $F$ and $\widetilde{F}$ stand for the chronological and antichronological Green functions, respectively, for magnetized vacuum, which are calculated by the Schwinger's proper time method as shown in the previous sections.

Setting Eqs. (35)-(37) into Eq. (34), we finally obtain the retarded Green function in terms of the chronological and antichronological Green functions for vacuum as 


$$
\begin{aligned}
i \widetilde{\Pi}_{r}\left(p_{0}\right)= & \frac{e^{2}}{2} \int \frac{d q_{0}}{2 \pi}\left[1-2 f\left(q_{0}+p_{0}\right)\right] \operatorname{Tr}\left[\Theta\left(-q_{0}\right) \gamma^{\mu} \widetilde{G}_{F}\left(q_{0}+p_{0}\right) \gamma^{\nu} \widetilde{G}_{F}\left(q_{0}\right)+\Theta\left(-q_{0}\right) \gamma^{\mu} \widetilde{G}_{\widetilde{F}}\left(q_{0}+p_{0}\right) \gamma^{\nu} \widetilde{G}_{F}\left(q_{0}\right)\right. \\
& \left.-\Theta\left(q_{0}\right) \gamma^{\mu} \widetilde{G}_{F}\left(q_{0}+p_{0}\right) \gamma^{\nu} \widetilde{G}_{\widetilde{F}}\left(q_{0}\right)-\Theta\left(q_{0}\right) \gamma^{\mu} \widetilde{G}_{\widetilde{F}}\left(q_{0}+p_{0}\right) \gamma^{\nu} \widetilde{G}_{\widetilde{F}}\left(q_{0}\right)\right]\left[\Theta\left(q_{0}+p_{0}\right)-\Theta\left(-q_{0}-p_{0}\right)\right] \\
& +\frac{e^{2}}{2} \int \frac{d q_{0}}{2 \pi}\left[1-2 f\left(q_{0}-p_{0}\right)\right] \operatorname{Tr}\left[\Theta\left(q_{0}\right) \gamma^{\mu} \widetilde{G}_{F}\left(q_{0}\right) \gamma^{\nu} \widetilde{G}_{F}\left(q_{0}-p_{0}\right)+\Theta\left(q_{0}\right) \gamma^{\mu} \widetilde{G}_{F}\left(q_{0}\right) \gamma^{\nu} \widetilde{G}_{\widetilde{F}}\left(q_{0}-p_{0}\right)\right. \\
& \left.-\Theta\left(-q_{0}\right) \gamma^{\mu} \widetilde{G}_{\widetilde{F}}\left(q_{0}\right) \gamma^{\nu} \widetilde{G}_{F}\left(q_{0}-p_{0}\right)-\Theta\left(-q_{0}\right) \gamma^{\mu} \widetilde{G}_{\widetilde{F}}\left(q_{0}\right) \gamma^{\nu} \widetilde{G}_{\widetilde{F}}\left(q_{0}-p_{0}\right)\right]\left[\Theta\left(q_{0}-p_{0}\right)-\Theta\left(-q_{0}+p_{0}\right)\right] .
\end{aligned}
$$

One easily recognizes that the structure of the integrand is quite similar to the vacuum polarization tensor apart from the integration over $q_{0}$ and the various combinations of $\widetilde{G}_{F}$ and $\widetilde{G}_{\tilde{F}}$. This enables us to simplify the integrand along the same line as for the vacuum case. Note that there is no summation over the Landau levels.

Denoting as ${ }^{F F} \Pi_{r}$ the contribution from the terms containing the product $G_{F} G_{F}$ and similarly for the other contributions, we calculate separately those terms. The antichronological Green function $G_{\tilde{F}}$ is obtained by taking the integration region $[-\infty, 0]$ instead of $[0, \infty]$ in the Schwinger proper time formalism:

$$
\begin{aligned}
G_{\widetilde{F}}\left(x, x^{\prime}\right)= & \frac{1}{i} \int_{-\infty}^{0} d s \exp \left\{-i\left[m^{2}-\left(\gamma^{\mu} \Pi_{\mu}\right)^{2}\right] s\right\} \\
& \times\left(\gamma^{\mu} \Pi_{\mu}+m\right) .
\end{aligned}
$$

Following Melrose and Stoneham [13], we can simplify this equation. Assuming that the vector potential is time independent and $A_{0}=0$, we can Fourier transform $G_{F}$ and $G_{\tilde{F}}$ with respect to $t-t^{\prime}$. Plugging them into the definition of the polarization tensor, one sees that the gauge-dependent terms cancel out just like the vacuum case and the polarization tensor becomes the function of the difference of the spatial coordinates, which then makes it possible for us to take the Fourier transformation with respect to the spatial coordinates. We finally obtain, for the contribution from the terms with the product $G_{F} G_{F}$,

$$
\begin{aligned}
& { }^{F F} \Pi_{r}^{\mu \nu}(p) \\
& \quad \int_{-\infty}^{\infty} \frac{d k_{0}}{8 \pi m} F F\left(k_{0}, p_{0}\right) \frac{e^{2} m^{2}}{(2 \pi)^{2}}(1-i) \sqrt{\frac{2 \pi}{L}} \int_{0}^{\infty} \frac{d \alpha}{\sqrt{\alpha}} \\
& \quad \times \int_{-\alpha}^{\alpha} d \beta \exp \left[-i \frac{\alpha}{L}\right] \exp \left[-i \frac{\alpha^{2}-\beta^{2}}{4 \alpha L} \frac{k_{z}^{2}}{m^{2}}\right] \\
& \quad \times \exp \left[-i \frac{\cos \beta-\cos \alpha}{2 L \sin \alpha} \frac{k_{\perp}^{2}}{m^{2}}\right] \\
& \quad \times \exp \left[i\left\{\frac{\alpha+\beta}{2 L} \frac{\left(k_{0}+p_{0}\right)^{2}}{m^{2}}+\frac{\alpha-\beta}{2 L} \frac{k_{0}^{2}}{m^{2}}\right\}\right] \times d^{\mu \nu} .
\end{aligned}
$$

Here $F F\left(k_{0}, p_{0}\right)$ is an abbreviation for the following function:

$$
\begin{aligned}
F F\left(k_{0}, p_{0}\right)= & \Theta\left(-k_{0}\right)\left[\Theta\left(k_{0}+p_{0}\right)-\Theta\left(-k_{0}-p_{0}\right)\right] \\
& \times\left[1-2 f\left(k_{0}+p_{0}\right)\right]+\Theta\left(k_{0}+p_{0}\right)\left[\Theta\left(k_{0}\right)\right. \\
& \left.-\Theta\left(-k_{0}\right)\right]\left[1-2 f\left(k_{0}\right)\right], \\
= & \Theta\left(k_{0}+p_{0}\right) \Theta\left(k_{0}\right)\left[1-2 f_{e}\left(k_{0}\right)\right]+\Theta\left(-k_{0}\right. \\
& \left.-p_{0}\right) \Theta\left(-k_{0}\right)\left[1-2 f_{e}+\left(\left|k_{0}+p_{0}\right|\right)\right] \\
& +\Theta\left(k_{0}+p_{0}\right) \Theta\left(-k_{0}\right)\left\{\left[1-2 f_{e}\left(k_{0}+p_{0}\right)\right]\right. \\
& \left.+\left[1-2 f_{e^{+}}\left(\left|k_{0}\right|\right)\right]\right\},
\end{aligned}
$$

where $f_{e}$ and $f_{e^{+}}$are Fermi-Dirac distribution functions for electron and positron, respectively. Except for the integral over the distribution functions, the resemblance of Eq. (40) to the vacuum counterpart is clear. The remaining factor $d^{\mu \nu}$, which is symmetric with respect to the superscripts, is given as

$$
\begin{aligned}
d^{01}= & -\frac{1}{2} \frac{\cos \beta-\cos \alpha}{\sin \alpha}\left\{\left[\cot \left(\frac{\alpha-\beta}{2}\right)-\cot \alpha\right]\right. \\
& \times \frac{k_{0}+p_{0}}{m}\left[\cot \left(\frac{\alpha+\beta}{2}\right) \frac{p_{x}}{m}\right]+\left[\cot \left(\frac{\alpha+\beta}{2}\right)-\cot \alpha\right] \\
& \left.\times \frac{k_{0}}{m}\left[\cot \left(\frac{\alpha-\beta}{2}\right) \frac{p_{x}}{m}\right]\right\} \\
d^{02}= & -\frac{1}{2} \frac{\cos \beta-\cos \alpha}{\sin \alpha}\left\{-\left[\cot \left(\frac{\alpha-\beta}{2}\right)-\cot \alpha\right]\right. \\
& \left.\times \frac{k_{0}+p_{0}}{m} \frac{p_{x}}{m}+\left[\cot \left(\frac{\alpha+\beta}{2}\right)-\cot \alpha\right] \frac{k_{0}}{m} \frac{p_{x}}{m}\right\} \\
d^{03}= & -\frac{1}{2} \cot \alpha \frac{p_{z}}{m}\left[2 \frac{k_{0}+p_{0}}{m}-\frac{p_{0}}{m}\left(1-\frac{\beta}{\alpha}\right)\right] \\
d^{13}= & -\frac{1}{2} \frac{\cos \beta}{\sin \alpha}\left[1-\frac{\beta}{\alpha} \frac{\tan \beta}{\tan \alpha} \frac{p_{x}}{m} \frac{p_{z}}{m}\right.
\end{aligned}
$$




$$
\begin{aligned}
d^{00}= & -\cot \alpha\left[\frac{k_{0}+p_{0}}{m} \frac{k_{0}}{m}-1+\frac{L}{2 \alpha} i+\frac{\alpha^{2}-\beta^{2}}{4 \alpha^{2}} \frac{p_{z}^{2}}{m^{2}}\right] \\
& +\frac{L}{\sin ^{2} \alpha} i-\frac{1}{2} \frac{\cos \beta-\cos \alpha}{\sin ^{3} \alpha} \frac{p_{x}^{2}}{m^{2}}, \\
d^{11}= & -\frac{\cos \beta}{\sin \alpha}\left[\frac{k_{0}+p_{0}}{m} \frac{k_{0}}{m}+1-\frac{L}{2 \alpha} i-\frac{\alpha^{2}-\beta^{2}}{4 \alpha^{2}} \frac{p_{z}^{2}}{m^{2}}\right] \\
& -\frac{1}{2} \frac{\cos \beta-\cos \alpha}{\sin ^{3} \alpha} \frac{p_{x}^{2}}{m^{2}}, \\
d^{22}= & -\frac{\cos \beta}{\sin \alpha}\left[\frac{k_{0}+p_{0}}{m} \frac{k_{0}}{m}+1-\frac{L}{2 \alpha} i-\frac{\alpha^{2}-\beta^{2}}{4 \alpha^{2}} \frac{p_{z}^{2}}{m^{2}}\right] \\
& +\frac{1}{2} \frac{\cos \beta-\cos \alpha}{\sin ^{3} \alpha} \frac{p_{x}^{2}}{m^{2}}, \\
d^{33}= & -\cot \alpha\left[\frac{k_{0}+p_{0}}{m} \frac{k_{0}}{m}+1+\frac{L}{2 \alpha} i+\frac{\alpha^{2}-\beta^{2}}{4 \alpha^{2}} \frac{p_{z}^{2}}{m^{2}}\right] \\
& -\frac{L}{\sin ^{2} \alpha} i+\frac{1}{2} \frac{\cos \beta-\cos \alpha}{\sin ^{3} \alpha} \frac{p_{x}^{2}}{m^{2}} .
\end{aligned}
$$

In deriving the above equation, we assumed $p^{\mu}$ $=\left(p_{0}, p_{x}, 0, p_{z}\right)$ and used the relation

$$
p_{\mu} \widetilde{\Pi}_{r}^{\mu \nu}(p)=\widetilde{\Pi}_{r}^{\mu \nu}(p) p_{\nu}=0,
$$

which is guaranteed for the current approximation [26].

The other contributions to $\Pi_{r}$ with different combinations of $G_{F}$ and $G_{\tilde{F}}$ are obtained in the same way. It turns out that the resultant equations are obtained from Eq. (40) with changes of the integral region and replacements of the distribution functions as shown below:

$$
\begin{aligned}
{ }^{F \widetilde{F}} \Pi_{r}^{\mu \nu}: F F\left(k_{0}, p_{0}\right) & \rightarrow F \widetilde{F}\left(k_{0}, p_{0}\right), \\
\text { integral region } & \rightarrow \int_{-\infty}^{\infty} d \alpha \int_{\alpha}^{\infty} d \beta, \\
\widetilde{F} F \Pi_{r}^{\mu \nu}: F F\left(k_{0}, p_{0}\right) & \rightarrow \widetilde{F} F\left(k_{0}, p_{0}\right), \\
\text { integral region } & \rightarrow \int_{-\infty}^{\infty} d \alpha \int_{-\infty}^{-\alpha} d \beta, \\
\widetilde{F} \widetilde{F} \Pi_{r}^{\mu \nu}: F F\left(k_{0}, p_{0}\right) & \rightarrow \widetilde{F} \widetilde{F}\left(k_{0}, p_{0}\right), \\
\text { integral region } & \rightarrow \int_{-\infty}^{0} d \alpha \int_{-\alpha}^{\alpha} d \beta .
\end{aligned}
$$

In the above equations, the phase should be taken as $\sqrt{-\alpha}$ $=-i \sqrt{|\alpha|}$, and the factors involving distribution functions are given as

$$
\begin{aligned}
F \widetilde{F}\left(k_{0}, p_{0}\right)= & -\Theta\left(k_{0}\right)\left[\Theta\left(k_{0}+p_{0}\right)-\Theta\left(-k_{0}-p_{0}\right)\right] \\
& \times\left[1-2 f\left(k_{0}+p_{0}\right)\right]+\Theta\left(k_{0}+p_{0}\right)\left[\Theta\left(k_{0}\right)-\Theta\right. \\
& \left.\left(-k_{0}\right)\right]\left[1-2 f\left(k_{0}\right)\right], \\
\widetilde{F} F\left(k_{0}, p_{0}\right)= & \Theta\left(-k_{0}\right)\left[\Theta\left(k_{0}+p_{0}\right)-\Theta\left(-k_{0}-p_{0}\right)\right] \\
& \times\left[1-2 f\left(k_{0}+p_{0}\right)\right]-\Theta\left(-k_{0}-p_{0}\right)\left[\Theta\left(k_{0}\right)\right. \\
& \left.-\Theta\left(-k_{0}\right)\right]\left[1-2 f\left(k_{0}\right)\right], \\
\widetilde{F} \widetilde{F}\left(k_{0}, p_{0}\right)= & -\Theta\left(k_{0}\right)\left[\Theta\left(k_{0}+p_{0}\right)-\Theta\left(-k_{0}-p_{0}\right)\right] \\
& \times\left[1-2 f\left(k_{0}+p_{0}\right)\right]-\Theta\left(-k_{0}-p_{0}\right)\left[\Theta\left(k_{0}\right)\right. \\
& \left.-\Theta\left(-k_{0}\right)\right]\left[1-2 f\left(k_{0}\right)\right] .
\end{aligned}
$$

Thus the polarization tensor is given by the sum of these terms

$$
\widetilde{\Pi}_{r}^{\mu \nu}={ }^{F F} \Pi_{r}^{\mu \nu}+{ }^{F}{ }^{\tilde{F}} \Pi_{r}^{\mu \nu}+{ }^{\tilde{F} F} \Pi_{r}^{\mu \nu}+{ }^{\tilde{F}} \widetilde{F} \Pi_{r}^{\mu \nu} .
$$

In the above equation, the "retardedness" of the polarization function is expressed by the combinations of the distribution functions of electron and positron. The analogous equation can be obtained for the advanced polarization tensor, which has different combinations of the distributions functions. It is noteworthy that the expression is symmetric with respect to the tensor indices unlike the imaginary time formalism where the antisymmetric terms arise as an imaginary part of the polarization tensor. This feature is taken over by the chronological polarization tensor in the real time formalism $[20,21]$.

It can be shown that the above formulas reduce to the vacuum polarization tensor employed in Sec. III in the limit of $T \rightarrow 0, \mu_{e} \rightarrow 0$. Here $\mu_{e}$ is the chemical potential of the electron. In fact, the chronological polarization tensor in this limit is obtained from

$$
\widetilde{\Pi}_{F}^{0}\left(p_{0}\right)=\Theta\left(p_{0}\right) \widetilde{\Pi}_{r}^{0}+\Theta\left(-p_{0}\right) \widetilde{\Pi}_{a}^{0} .
$$

The superscript " 0 " means the polarization tensors for vacuum. Inserting Eq. (33) and the corresponding expression for the advanced polarization tensor

$$
\begin{aligned}
i \Pi_{a}^{\mu \nu}\left(x, x^{\prime}\right)= & \frac{e^{2}}{2} \operatorname{Tr}\left[\gamma^{\mu} G_{c}\left(x, x^{\prime}\right) \gamma^{\nu} G_{r}\left(x^{\prime}, x\right)\right. \\
& \left.+\gamma^{\mu} G_{a}\left(x, x^{\prime}\right) \gamma^{\nu} G_{c}\left(x^{\prime}, x\right)\right],
\end{aligned}
$$

and using the fact that the combinations like $G_{r} G_{r}$ and $G_{a} G_{a}$ vanish after integration over energy, we can show that only the right combination $G_{F} G_{F}$ remains.

It is more convenient to take a projection of the above result onto the basis tensors constructed from $F_{\mu \nu}, F_{\mu \nu}^{*}$, $p_{\mu}$, and $u_{\mu}$. Here $F_{\mu \nu}^{*}$ is the dual tensor of $F_{\mu \nu}$ and $u_{\mu}$ is the four velocity of the plasma. Following Rojas and Shabad $[23,24]$, we take as the basis tensors

$$
\Psi_{\mu \nu}^{(1)}=p^{2} g_{\mu \nu}-p_{\mu} p_{\nu},
$$


$\Psi_{\mu \nu}^{(2)}=\left[F_{\mu \lambda} p^{\lambda}\right]\left[F_{\nu \sigma} p^{\sigma}\right]$,

$\Psi_{\mu \nu}^{(3)}=-\left[p^{2} g_{\mu \lambda}-p_{\mu} p_{\lambda}\right]\left[\frac{\left(F^{2}\right)^{\lambda \sigma}}{p^{2}}\right]\left[p^{2} g_{\sigma \nu}-p_{\sigma} p_{\nu}\right]$,

$$
\begin{aligned}
\Psi_{\mu \nu}^{(4)}= & \left\{\left[p^{2}\left(F^{2} p\right)_{\mu}-\left(p F^{2} p\right) p_{\mu}\right]\left[F_{\nu \lambda}^{*} p^{\lambda}\right]+\left[F_{\mu \sigma}^{*} p^{\sigma}\right]\right. \\
& \times\left[p^{2}\left(F^{2} p\right)_{\nu}\right. \\
& \left.\left.-\left(p F^{2} p\right) p_{\nu}\right]\right\} /\left\{\left[p^{2}\left(p F^{2} p\right)\right]^{1 / 2}\left[p\left(F^{*}\right)^{2} p\right]\right\},
\end{aligned}
$$$$
\Psi_{\mu \nu}^{(7)}=\left[p^{2}\left(F^{2} p\right)_{\mu}-\left(p F^{2} p\right) p_{\mu}\right]\left[F_{\nu \lambda} p^{\lambda}\right]+\left[F_{\mu \sigma} p^{\sigma}\right]
$$$$
\times\left[p^{2}\left(F^{2} p\right)_{\nu}-\left(p F^{2} p\right) p_{\nu}\right],
$$

$$
\begin{aligned}
\Psi_{\mu \nu}^{(8)}= & \left(u_{\rho} p^{\rho}\right)\left[\left(u_{\mu}-\frac{p_{\mu}\left(u_{\rho} p^{\rho}\right)}{p^{2}}\right)\left[F_{\nu \lambda} p^{\lambda}\right]+\left[F_{\mu \sigma} p^{\sigma}\right]\right. \\
& \left.\times\left(u_{\mu}-\frac{p_{\mu}\left(u_{\rho} p^{\rho}\right)}{p^{2}}\right)\right] .
\end{aligned}
$$

In the above equations $g_{\mu \nu}=(1,-1,-1,-1)$ is the metric, and $p^{2}=p_{\mu} p^{\mu},\left(F^{2}\right)_{\mu \nu}=F_{\mu \rho} F_{\nu}^{\rho}$. The polarization tensor is expanded with these basis tensors as

$$
\widetilde{\Pi}_{r}^{\mu \nu}(p)=\sum_{i} \pi^{i} \Psi^{(i) \mu \nu}
$$

The coefficients are easily calculated by taking projections on the orthogonal basis vectors $[23,24]$ defined as

$$
\begin{aligned}
& e_{\mu}^{(1)}=\frac{p^{2}\left(F^{2} p\right)_{\mu}-\left(p F^{2} p\right) p_{\mu}}{\left[p^{2}\left(p F^{2} p\right)\left(p F^{* 2} p\right)\right]^{1 / 2}}, \\
& e_{\mu}^{(2)}=\frac{F_{\mu \lambda}^{*} p^{\lambda}}{\left[p F^{* 2} p\right]^{1 / 2}}, \\
& e_{\mu}^{(3)}=\frac{F_{\mu \lambda} p^{\lambda}}{\left[p F^{2} p\right]^{1 / 2}}, \\
& e_{\mu}^{(4)}=\frac{p_{\mu}}{\left[p^{2}\right]^{1 / 2}} .
\end{aligned}
$$

We expand $d^{\mu \nu}$ in Eq. (40) according to Eq. (67) as

$$
d^{\mu \nu}=\sum_{i} d^{i} \Psi^{(i) \mu \nu}
$$

In Eq. (72), it should be understood that only the nonvanishing part after integration is expanded. Defining further

$$
a_{1} \equiv e_{\mu}^{(1)} d^{\mu \nu} e_{\nu}^{(1)}
$$

$$
\begin{aligned}
= & \frac{p_{z}^{2} p_{x}}{p_{0}\left(p_{0}^{2}-p_{z}^{2}\right)} d^{01}+\frac{p_{0}^{2}-p_{z}^{2}}{p_{0} p_{z}} d^{03} \\
& -\frac{p_{0}^{2} p_{x}}{p^{2}\left(p_{0}^{2}-p_{z}^{2}\right)} d^{13}
\end{aligned}
$$

$$
\begin{aligned}
a_{2} \equiv & e_{\mu}^{(2)} d^{\mu \nu} e_{\nu}^{(2)} \\
= & \frac{p_{z}^{2} p_{x}}{p_{0}\left(p_{0}^{2}-p_{z}^{2}\right)} d^{01}+\frac{p_{0}^{2}-p_{z}^{2}}{p_{0} p_{z}} d^{03} \\
& -\frac{p_{0}^{2} p_{x}}{p_{z}\left(p_{0}^{2}-p_{z}^{2}\right)} d^{13},
\end{aligned}
$$

$$
\begin{aligned}
a_{3} \equiv & e_{\mu}^{(3)} d^{\mu \nu} e_{\nu}^{(3)} \\
= & \frac{p_{0}}{p_{x}} d^{01}-\frac{p_{z}}{p_{x}} d^{13} \\
& +\frac{\cos \beta-\cos \alpha}{\sin ^{3} \alpha} \frac{p_{x}^{2}}{m^{2}}, \\
a_{4} \equiv & e_{\mu}^{(2)} d^{\mu \nu} e_{\nu}^{(1)} \\
= & \frac{\sqrt{p^{2}}}{p_{0}^{2}-p_{z}^{2}}\left[p_{z} d^{01}-p_{0} d^{13}\right],
\end{aligned}
$$

$$
\begin{aligned}
a_{5} & \equiv e_{\mu}^{(3)} d^{\mu \nu} e_{\nu}^{(1)} \\
& =-\frac{p_{0} p_{x}}{\sqrt{p^{2}\left(p_{0}^{2}-p_{z}^{2}\right)}} d^{02}, \\
a_{6} & \equiv e_{\mu}^{(2)} d^{\mu \nu} e_{\nu}^{(3)} \\
& =\frac{p_{z}}{\sqrt{\left(p_{0}^{2}-p_{z}^{2}\right)}} d^{02},
\end{aligned}
$$

we can express the coefficients $d^{i}$ in terms of $a_{i}$ as

$$
d^{1}=-\frac{a_{2}}{p^{2}}
$$

$d^{2}=\frac{1}{B^{2} p_{0}^{2}}\left[a_{3}-a_{2}-\frac{p^{2}}{p_{0}^{2}-p_{z}^{2}}\left(a_{1}-a_{2}\right)\right]$,

$d^{3}=-\frac{a_{1}-a_{2}}{B^{2}\left(p_{0}^{2}-p_{z}^{2}\right)}$,

$d^{4}=a_{4}$, 
$d^{7}=\frac{1}{B p_{0} p_{x}} \sqrt{\frac{1}{p^{2}\left(p_{0}^{2}-p_{z}^{2}\right)}} a_{5}+\frac{p_{0}}{B^{3} p^{2} p_{x} p_{z}} \sqrt{\frac{1}{\left(p_{0}^{2}-p_{z}^{2}\right)}} a_{6}$,

$d^{8}=-\frac{1}{B p_{0} p_{x}} \sqrt{\frac{p_{0}^{2}-p_{z}^{2}}{p_{z}^{2}}} a_{6}$.

Combining the above equations with Eqs. (43)-(50), we finally obtain $\pi^{i}$ in Eq. (67).

Since the medium corrections occur only for on-shell momenta, they do not induce extra divergence to regularize. Hence we have only to renormalize a vacuum contribution. This is simply done by subtracting the unrenormalized vacuum part and then adding the renormalized vacuum polarization tensor:

$$
\operatorname{reg} \Pi=\Pi-\left.\Pi\right|_{T=\mu_{e}=0}+\left.\operatorname{reg} \Pi\right|_{T=\mu_{e}=0} .
$$

In the above equation, "reg" implies the regularized tensors. It is noted that reg $\left.\Pi\right|_{T=\mu_{e}=0}$ is nothing but the tensor calculated in the preceding sections. It is also possible to subtract $\left.\Pi\right|_{T=\mu_{e}=B=0}$ and to add Eq. (14). Only $\pi^{1}$ should be renormalized as in the vacuum case.

As mentioned already, our formalism is closely related to that obtained in $[20,21]$. Both are based on the one-loop approximation and utilize the chronological and antichronological propagators of electron in vacuum. Hence it is obvious that they contain essentially the same contents. This is explicitly shown as follows. It is known that the exact polarization tensors are related by

$$
\Pi^{11}\left(p_{0}\right)=\Pi_{r}\left(p_{0}\right)\left[1+n\left(p_{0}\right)\right]-\Pi_{a}\left(p_{0}\right) n\left(p_{0}\right),
$$

where $\widetilde{\Pi}^{11}\left(p_{0}\right)$ is the (11)-component or the chronological component of the $2 \times 2$ matrix polarization tensor in the real time formalism, and $n\left(p_{0}\right)$ is the Bose distribution function for the electron positron pair. Applying this relation to the one-loop approximation given by Eq. (34) and the corresponding equation for $\widetilde{\Pi}_{a}\left(p_{0}\right)$ obtained by exchanging $\widetilde{G}_{r}$ and $\widetilde{G}_{a}$ in Eq. (34), we obtain $\widetilde{\Pi}^{11}\left(p_{0}\right)$ expressed in terms of $\widetilde{G}_{r}$ and $\widetilde{G}_{a}$ after using the relation Eq. (35). Inserting the approximations Eqs. (36) and (37) valid for the ideal electron, we get $\widetilde{\Pi}^{11}\left(p_{0}\right)$ expressed in terms of $\widetilde{G}_{F}$ and $\widetilde{G}_{\widetilde{F}}$. It is easy to show using the relation

$$
f\left(q_{0}+p_{0}\right)-f\left(q_{0}\right)=-\frac{1}{n\left(p_{0}\right)} f\left(q_{0}+p_{0}\right)[1-f(q)]
$$

that the final expression becomes

$$
i \widetilde{\Pi}^{11}\left(p_{0}\right)=e^{2} \int \frac{d q_{0}}{2 \pi} \operatorname{Tr}\left[\gamma^{\mu} \widetilde{G}^{11}\left(q_{0}+p_{0}\right) \gamma^{\nu} \widetilde{G}^{11}\left(q_{0}\right)\right] .
$$

Here the (11)-component of the electron's Green function in the ideal magnetic plasma is given in terms of $\widetilde{G}_{F}$ and $\widetilde{G}_{\tilde{F}}$ as

$$
\begin{aligned}
\widetilde{G}^{11}\left(q_{0}\right) & =\widetilde{G}_{F}\left(q_{0}\right)-\eta_{F}\left(q_{0}\right)\left[\widetilde{G}_{F}\left(q_{0}\right)-\widetilde{G}_{\widetilde{F}}\left(q_{0}\right)\right], \\
\eta_{F}\left(q_{0}\right) & =\Theta\left(q_{0}\right) f\left(q_{0}\right)+\Theta\left(-q_{0}\right)\left[1-f\left(q_{0}\right)\right] .
\end{aligned}
$$

This is exactly the same expression for $\widetilde{\Pi}^{11}$ derived in $[20,21]$. The above manipulation clearly shows that $\widetilde{\Pi}^{11}$ in $[20,21]$ and $\tilde{\Pi}_{r}\left(p_{0}\right)$ in this paper are related in the same way as the exact polarization tensors and that the difference lies only in their imaginary parts. Hence the results obtained in $[20,21]$ for the weak magnetic field should hold for our polarization tensor, since it contains the same physics. Our expression is slightly closer to the vacuum polarization tensor employed in the previous sections. The main aim of this section is to show that the reduction of the fivefold integration obtained in $[20,21]$ can be done in quite a similar way to the vacuum case and that the resulting expression becomes also similar to the vacuum counterpart.

Although the form of $d^{\mu \nu}$ in Eqs. (43)-(50) is very similar to the vacuum counterpart, the numerical evaluation of them is very difficult. This is so for the same reason as for the numerical evaluation of the vacuum polarization tensor above the threshold of the pair creation. This prevents us from performing a Wick rotation for the $\alpha$ and $\beta$ integrations in Eq. (40). These should be the next steps.

\section{DISCUSSIONS}

We have numerically computed the vacuum polarization tensor by using Schwinger's proper-time method for various strengths of the background magnetic field, photon energies, and angle of propagation below the threshold of the pair creation. One advantage of the formulation employed here is the fact that we do not have to perform the summations over the discrete Landau levels up to infinity but have only to do the integrations over proper time. We then find that we can handle the infinite integral by appropriate conversion of variables. In this paper, one of our main purposes is the numerical evaluations of the polarization tensors and to serve as a bridge between the analytic formulas given so far for some limiting cases. It is noted again that the explicit numerical values near the threshold energy of the pair creation were absent in the literatures. In addition, we have obtained the fitting formula which reproduces the numerical results within $10 \%$ of error. In the case of the low energy and strong or weak magnetic-field limits, it exactly agrees with the analytical estimations. We hope this formula will help astrophysicists build the astrophysical models.

It is true that the current results are confined below the threshold of pair creation and that the behavior above the threshold is very important. However, this region still contains interesting parameter regions from the point of view of the astrophysics, e.g., x-ray observations ( $E=$ a few $\mathrm{keV}$ $-100 \mathrm{keV})$. Provided the discovery of the magnetars, it is not unreasonable to expect other extraterrestrial objects which have even larger magnetic fields to be found in the future observations. In such superstrong magnetic fields, we expect that nontrivial lensing effects in x-rays would occur due to the modifications of the refractive indices. Then, as- 
trophysicists should consider such lensing effects there for $\mathrm{x}$-ray observations. Therefore, we think our work would be helpful and useful for such observations of $\mathrm{x}$ rays.

So far, we have had a foreboding that the polarization tensor would have nontrivial behaviors above the threshold energy. The numerical study above the threshold is certainly our next step and is indeed currently underway [19].

We have also presented the expression of the retarded polarization tensor for the finite density and temperature in the one-loop approximation, utilizing the chronological and antichronological vacuum Green function in vacuum. It is shown that the multiple integrations are simplified in an analogous way to the vacuum case. It is shown the expression is reduced to the vacuum one in the limit of zero temperature and density. It is also demonstrated that the present formulation contains the same contents as that derived in $[20,21]$ and, therefore, that their results for weak magnetic fields are true for our case. The direct confirmation will be done when the numerical evaluations of the expression are performed [19]. Our concern, however, is rather directed to the very strong magnetic field where no analytic evaluation is expected. It is emphasized that the remaining issue for that purpose is common to the numerical evaluations of the vacuum polarization tensor above the threshold energy of pair creations.

Our final goal is to calculate not only the polarization tensor but also other physical quantities under strong magnetic fields such as the photon splitting rates and equation of states for a wide range of parameters. This paper is the first step of this project.

\section{ACKNOWLEDGMENTS}

This work is partially supported by the Grants-in-Aid by the Ministry of Education, Science, Sports and Culture of Japan (No. 12740138).
[1] R.C. Duncan, in Proceedings of Fifth Huntsville Gamma-Ray Burst Symposium, astro-ph/0002442.

[2] D. Lai, Rev. Mod. Phys. 73, 629 (2001).

[3] C. Kouveliotou et al., Nature (London) 393, 235 (1998).

[4] R.C. Duncan and C. Thompson, Astrophys. J. Lett. 392, L9 (1992).

[5] B. Paczyński, in The Largest Explosions Since the Big Bang: Supernovae and Gamma Ray Bursts, edited by M. Livio, K. Sahu, and N. Panagia (Cambridge University Press, Cambridge, England, 2001), astro-ph/9909048.

[6] R.D. Blandford and R.L. Znajek, Mon. Not. R. Astron. Soc. 179, 433 (1977).

[7] J.C. Wheeler, in The Largest Explosions Since the Big Bang: Supernovae and Gamma Ray Bursts, edited by M. Livio, K. Sahu, and N. Panagia (Cambridge University Press, Cambridge, 2001), astro-ph/9909096.

[8] E.M.D. Symbalisty, Astrophys. J. 285, 729 (1984).

[9] A. Kusenko and G. Segre, Phys. Rev. D 59, 061302(R) (1999).

[10] C.J. Horowitz and G. Li, Phys. Rev. D 61, 063002 (2000).

[11] S.L. Adler, Ann. Phys. (N.Y.) 67, 599 (1971).

[12] A.E. Shabad, Ann. Phys. (N.Y.) 90, 166 (1975).

[13] D.B. Melrose and R.J. Stoneham, Nuovo Cimento A 32, 435 (1976).
[14] P. Mészáros, High-Energy Radiation from Magnetized Neutron Stars (University of Chicago Press, Chicago, 1992).

[15] C. Thompson and R.D. Duncan, Mon. Not. R. Astron. Soc. 275, 255 (1995).

[16] T. Bulik and M.C. Miller, Mon. Not. R. Astron. Soc. 288, 596 (1997).

[17] J. Schwinger, Phys. Rev. 82, 664 (1951).

[18] V.I. Denisov, I.P. Denisova, and S.I. Svertilov, astro-ph/0110705.

[19] K. Kohri and S. Yamada (in preparation).

[20] A.K. Ganguly, S. Konar and P.S. Pal, Phys. Rev. D 60, 105014 (1999).

[21] A.K. Ganguly and S. Konar, Phys. Rev. D 63, 065001 (2001).

[22] J. Alexander, Phys. Rev. D 63, 073010 (2001).

[23] H. Pérez Rojas and A.E. Shabad, Ann. Phys. (N.Y.) 121, 432 (1979).

[24] H. Pérez Rojas and A.E. Shabad, Ann. Phys. (N.Y.) 138, 1 (1982).

[25] K.-C. Chou, Z.-B. Su, B.-L. Hao and L. Yu, Phys. Rep. 118, 1 (1985).

[26] Yu.B. Ivanov, J. Knoll, and D.N. Voskresensky, Nucl. Phys. A657, 413 (1999). 